\title{
Implementation of symmetrizers in ordinary differential equations
}

\author{
N. Razali ${ }^{1} \quad$ R.P.K. Chan ${ }^{2}$
}

(Received 18 December 2013; revised 10 July 2015)

\begin{abstract}
Two-step symmetrizers for the implicit midpoint and trapezoidal rules provide an alternative to the one-step smoothing formula for solving stiff ordinary differential equations. When used with the basic symmetric methods, these L-stable methods preserve the asymptotic error expansion in even powers of the step size and provide the necessary damping of oscillatory solutions. These new symmetrizers show effects similar to one-step smoothing but with the advantage of being order two. When generalized to higher order symmetric methods, such as the twostage Gauss or the three-stage Lobatto IIIA, these symmetrizers can suppress order reduction for stiff problems. Here, we discuss one-step and two-step symmetrizers and their application in ordinary differential equations. We present numerical results with constant and variable
\end{abstract}

http://journal.austms.org.au/ojs/index.php/ANZIAMJ/article/view/7833 gives this article, (c) Austral. Mathematical Soc. 2015. Published August 5, 2015, as part of the Proceedings of the 11th Biennial Engineering Mathematics and Applications Conference. ISSN 1446-8735. (Print two pages per sheet of paper.) Copies of this article must not be made otherwise available on the internet; instead link directly to this URL for this article. 
step sizes that show the advantages of two-step symmetrizers over one-step symmetrizers of the implicit trapezoidal rule for stiff linear and nonlinear problems.

\section{Contents}

1 Introduction

C542

2 Order analysis of the Prothero-Robinson problem

C544

2.1 One-step symmetrization of ITR . . . . . . . . . C546

2.2 Two-step symmetrization of ITR . . . . . . . . . C546

3 Implementation

C547

3.1 Problems using constant step size . . . . . . . . . . . C547

3.2 Problems using variable step sizes . . . . . . . . . . . C553

4 Results and discussion

C553

5 Conclusion

C558

References

C561

\section{Introduction}

Symmetric Runge-Kutta methods are implicit methods for solving stiff ordinary differential equations. These methods possess asymptotic error expansions in even powers of the step size [17] which are exploited with Richardson extrapolation [16] to increase the order of the method by two.

Gragg [11] proved the existence of an expansion for the explicit midpoint rule and introduced the concept of smoothing to suppress the parasitic oscillatory component in the numerical solution of nonstiff problems. The application of 
smoothing applied with extrapolation was investigated for nonstiff [2] and later for stiff problems [14, 7, 1].

Chan [3, 4] generalized the concept of smoothing to arbitrary symmetric RungeKutta methods, calling it symmetrization. This process is achieved by means of a related Runge-Kutta method called a symmetrizer. It is constructed so as to preserve symmetry (asymptotic $h^{2}$-error expansion in step size $h$ ) and to provide damping for stiff problems. It also has the advantage of suppressing order reduction when used with higher order methods. Chan and Razali [5] recently studied two-step symmetrization with constant step size for order two symmetric methods.

In this article, smoothing of the implicit trapezoidal rule (ITR) is extended to two-step symmetrization. This symmetrization is equivalent to a symmetrizer applied over two steps. The advantage of two-step symmetrization is that when it is applied in active mode, the method has order two behaviour compared to the order one behaviour of the one-step symmetrization [10]. The two-step symmetrization of the ITR also shows order four super-convergence for linear stiff Prothero-Robinson problems. The Butcher tableaus for the one-step and two-step symmetrizers are, respectively,

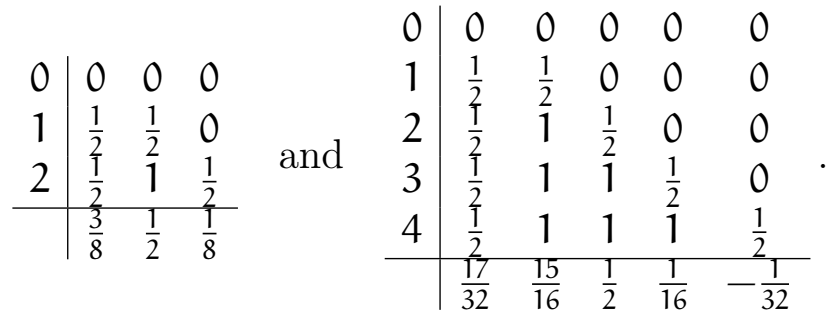

In general, we either apply symmetrization in the passive or active mode. In the passive mode we compute many steps with the symmetric method, store the update and internal stage values at each step and apply symmetrization whenever required. In the active mode we use the symmetrized value to propagate the numerical solution each time it is computed. We can also perform symmetrization at every step, every two steps, or a combination of these. 
In the constant step size setting, we are interested to see whether the superconvergent order four behaviour is preserved for other stiff linear problems. This is important, especially when we propose to apply extrapolation to achieve higher order behaviour. We also investigate the performance of twostep symmetrization with variable step size setting. Section 2 presents an analysis on the Prothero-Robinson problem and Section 3 implements the one-step and two-step symmetrizations of the implicit trapezoidal rule on linear and non-linear problems. Section 4 presents some results with constant and variable step size settings.

\section{Order analysis of the Prothero-Robinson problem}

An s-stage Runge-Kutta method applied to $y^{\prime}=f(x, y)$ with step size $h$ is defined by

$$
\begin{aligned}
& Y_{i}=y_{n-1}+h \sum_{j=1}^{s} a_{i j} f\left(x_{n-1}+c_{j} h, Y_{j}\right), \quad i=1, \ldots, s, \\
& y_{n}=y_{n-1}+h \sum_{i=1}^{s} b_{i} f\left(x_{n-1}+c_{i} h, Y_{i}\right),
\end{aligned}
$$

where $A=\left[a_{i j}\right]$ is the $s \times s$ Runge-Kutta matrix, and $b$ and $c$ are the $s$ dimensional vectors of weights and abscissas, respectively.

We analyse the Prothero-Robinson (PR) problem [15] where

$$
y^{\prime}(x)=\lambda[y(x)-g(x)]+g^{\prime}(x), \quad y\left(x_{0}\right)=g\left(x_{0}\right),
$$

with $\mathrm{g}$ a smooth function and stiffness parameter $\lambda \in \mathbb{C}$ with $\operatorname{Re}(\lambda)<0$. The problem becomes more stiff as $|\lambda|$ increases but has the same exact solution, independent of the stiffness parameter. Hairer and Wanner [12] calculated the 
global error of the numerical solution after $\boldsymbol{n}$ steps. In the case of a one-step symmetrization applied in active mode, the global error is

$$
\widetilde{\epsilon}_{\mathfrak{n}}=\widetilde{y}_{n}-y\left(x_{n}\right)=\sum_{i=1}^{n} \widetilde{R}(z)^{n-i} \widetilde{\psi}_{i}(z)
$$

where $\widetilde{R}(z)$ is the stability function of the symmetrizer with $z=\lambda \mathrm{h}$ and the local error for step $i$ is

$$
\widetilde{\psi}_{i}(z)=\sum_{k=2}^{\infty} \frac{h^{k}}{k !} y^{(k)}\left(x_{i-1}\right)\left[1-k \widetilde{b}^{\top} \widetilde{c}^{k-1}+z \widetilde{b}^{\top}(\widetilde{\mathrm{I}}-z \widetilde{A})^{-1}\left(\widetilde{c}^{k}-k \widetilde{A} \widetilde{c}^{k-1}\right)\right],
$$

where $(\widetilde{A}, \widetilde{b}, \widetilde{c})$ are the coefficients of the one-step symmetrizer and $\widetilde{I}$ is the identity matrix.

If one-step symmetrization is applied in passive mode at step $n$, then the global error is

$$
\widetilde{\epsilon}_{n}=\widetilde{R}(z) \epsilon_{n-1}+\widetilde{\psi}_{n}(z),
$$

where $\widetilde{\psi}_{n}$ is the local error at step $n$ and the stability function is

$$
\widetilde{R}(z)=\frac{1}{\left(1-\frac{1}{2} z\right)^{2}} .
$$

For two-step symmetrization applied in active mode, the global error has the same form as (4) but the local error for step $i$ is

$$
\widetilde{\psi}_{i}(z)=\sum_{k=2}^{\infty} \frac{h^{k}}{k !} y^{(k)}\left(x_{i-2}\right)\left[2^{k}-k \widetilde{b}^{T} \widetilde{c}^{k-1}+z \widetilde{b}^{\top}(\widetilde{I}-z \widetilde{A})^{-1}\left(\widetilde{c}^{k}-k \widetilde{A} \widetilde{c}^{k-1}\right)\right] \text {, }
$$

where $(\widetilde{A}, \widetilde{b}, \widetilde{c})$ are now the coefficients of the two-step symmetrizer.

If the two-step symmetrization is applied in passive mode, then the global error is

$$
\widetilde{\epsilon}_{n}=\widetilde{R}(z) \epsilon_{n-2}+\widetilde{\psi}_{n}(z)
$$


where $\widetilde{\psi}_{\mathfrak{n}}$ is the local error at step $\mathrm{n}$ and the stability function is

$$
\widetilde{\mathrm{R}}(z)=\frac{1-\frac{1}{2} z^{2}}{\left(1-\frac{1}{2} z\right)^{4}} .
$$

\subsection{One-step symmetrization of ITR}

One-step symmetrization of the ITR is

$$
\widetilde{y}_{n}=\frac{y_{n-1}+2 y_{n}+y_{n+1}}{4}
$$

and is the same as the smoothing formula introduced by Gragg [11]. We refer to the problem as nonstiff when $|\lambda| \sim \mathcal{O}(1)$ and strongly stiff when $1 /|\lambda| \sim \mathcal{O}\left(h^{2}\right)$. Substituting the coefficients of the one-step symmetrizer (1) into equations (5) and (6) we obtain the local and global errors as $h \rightarrow 0$ in passive mode

$$
\begin{aligned}
\widetilde{\psi}_{\mathfrak{n}}(z) & = \begin{cases}\mathcal{O}\left(h^{2}\right) & \text { if nonstiff, } \\
\mathcal{O}\left(h^{2}\right) & \text { if strongly stiff, }\end{cases} \\
\widetilde{\epsilon}_{\mathfrak{n}} & = \begin{cases}\mathcal{O}\left(h^{2}\right) & \text { if nonstiff, } \\
\mathcal{O}\left(h^{2}\right) & \text { if strongly stiff. }\end{cases}
\end{aligned}
$$

The global errors for one-step symmetrization in active mode as $h \rightarrow 0$ are

$$
\widetilde{\epsilon}_{n}= \begin{cases}\mathcal{O}(h) & \text { if nonstiff, } \\ \mathcal{O}\left(h^{2}\right) & \text { if strongly stiff. }\end{cases}
$$

\subsection{Two-step symmetrization of ITR}

Two-step symmetrization of ITR is

$$
\widetilde{y}_{n}=\frac{-y_{n-2}+4 y_{n-1}+10 y_{n}+4 y_{n+1}-y_{n+2}}{16} \text {. }
$$


Substituting the coefficients of the two-step symmetrizer (1) into equations (8) and (9) we obtain the local and global errors as $h \rightarrow 0$ in passive mode

$$
\begin{aligned}
\widetilde{\psi}_{n}(z) & = \begin{cases}\mathcal{O}\left(h^{3}\right) & \text { if nonstiff, } \\
\mathcal{O}\left(h^{3}\right) & \text { if strongly stiff, }\end{cases} \\
\widetilde{\epsilon}_{\mathfrak{n}} & = \begin{cases}\mathcal{O}\left(h^{2}\right) & \text { if nonstiff, } \\
\mathcal{O}\left(h^{2} / \lambda\right)=\mathcal{O}\left(h^{4}\right) & \text { if strongly stiff. }\end{cases}
\end{aligned}
$$

The global errors for two-step symmetrization of ITR as $h \rightarrow 0$ in active mode are

$$
\widetilde{\epsilon}_{n}= \begin{cases}\mathcal{O}\left(h^{2}\right) & \text { if nonstiff, } \\ \mathcal{O}\left(h^{2} / \lambda\right)=\mathcal{O}\left(h^{4}\right) & \text { if strongly stiff. }\end{cases}
$$

\section{Implementation}

In this section, we discuss the implementation of symmetrization using constant and variable step sizes. We investigate the accuracy and efficiency of one-step and two-step symmetrizations of the ITR. The costly part is computing the Newton iterations for the internal stage values. Thus, we use simplified Newton iterations by computing the Jacobian only on the first approximation instead of computing it at each iteration. We also use compensated summation in order to minimize round off error [12]. We denote one-step symmetrization applied in active and passive modes as 1AS and 1PS, respectively, while two-step symmetrization in active and passive modes are denoted as $2 \mathrm{AS}$ and 2PS, respectively.

\subsection{Problems using constant step size}

We consider the following constant step size problems. 
- The PR problem (3) integrated to $X=5$ with step size $h=0.1$ and $g(x)=\exp (-x)$.

- The Frank, Schneid and Ueberhuber (FSU) [9] problem

$$
y^{\prime}=\lambda y+e^{-x}, \quad y(0)=-\frac{1}{1+\lambda}, \quad \lambda=10^{6},
$$

integrated to $X=5$ with step size $h=0.1$.

- The Holsapple, Iyer and Doman (HID) [13] problem

$$
y^{\prime}=-\lambda y+\sin (x), \quad y(0)=-\frac{1}{1000001}, \quad \lambda=10^{3},
$$

integrated to $X=5$ with step size $h=0.5$.

The application of symmetrization with constant step size shows that two-step symmetrization in active mode is the most accurate method when used to solve the linear PR problem [15]. This is the result of the higher order of the method. The analysis on superconvergent order four behaviour of two-step symmetrization is verified numerically by application to stiff linear problems. The order is computed in Matlab from the slope of the log-log plot of absolute error versus step size.

In Figure 1 for the stiff PR with $\lambda=-1$, all methods are order two except $1 \mathrm{AS}$ which is order one. In Figure 2 for the nonstiff PR with $\lambda=-10^{6}, 2 \mathrm{AS}$ and 2PS are superconvergent order four. In Figure 3 for FSU, 2PS and 2AS are superconvergent order four and in Figure 4 for HID, 2PS and 2AS are also superconvergent order four. There are two methods with visually identical plots in all figures. In Figure 1, both the ITR and 2PS lie along the same line, while in Figure 2, both 1AS and 1PS lie along the same line. In Figures 3 and 4 for FSU and HID, 1AS and 1PS lie along the same line. 


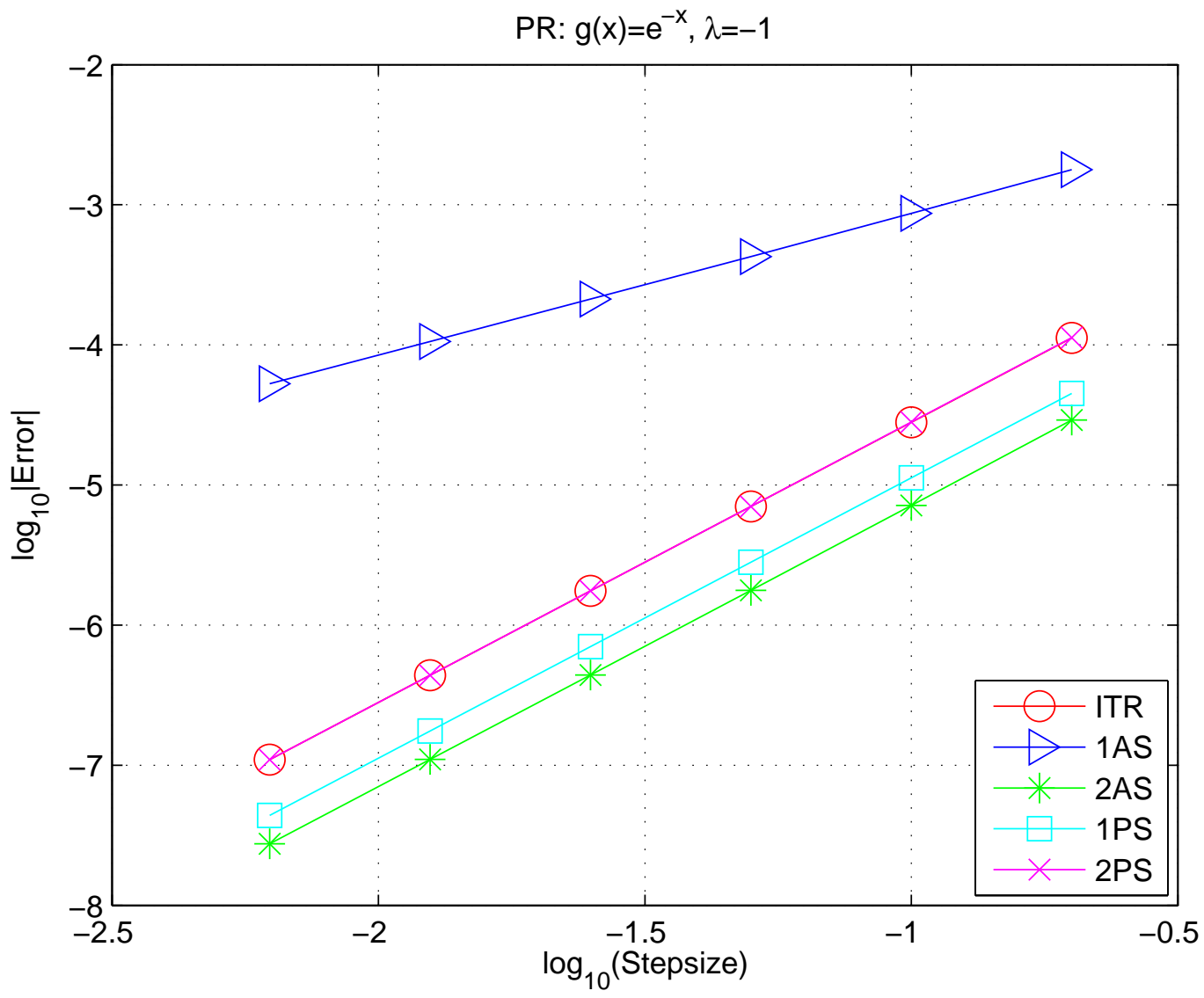

Figure 1: The PR problem order behaviour of the ITR for the nonstiff case. 


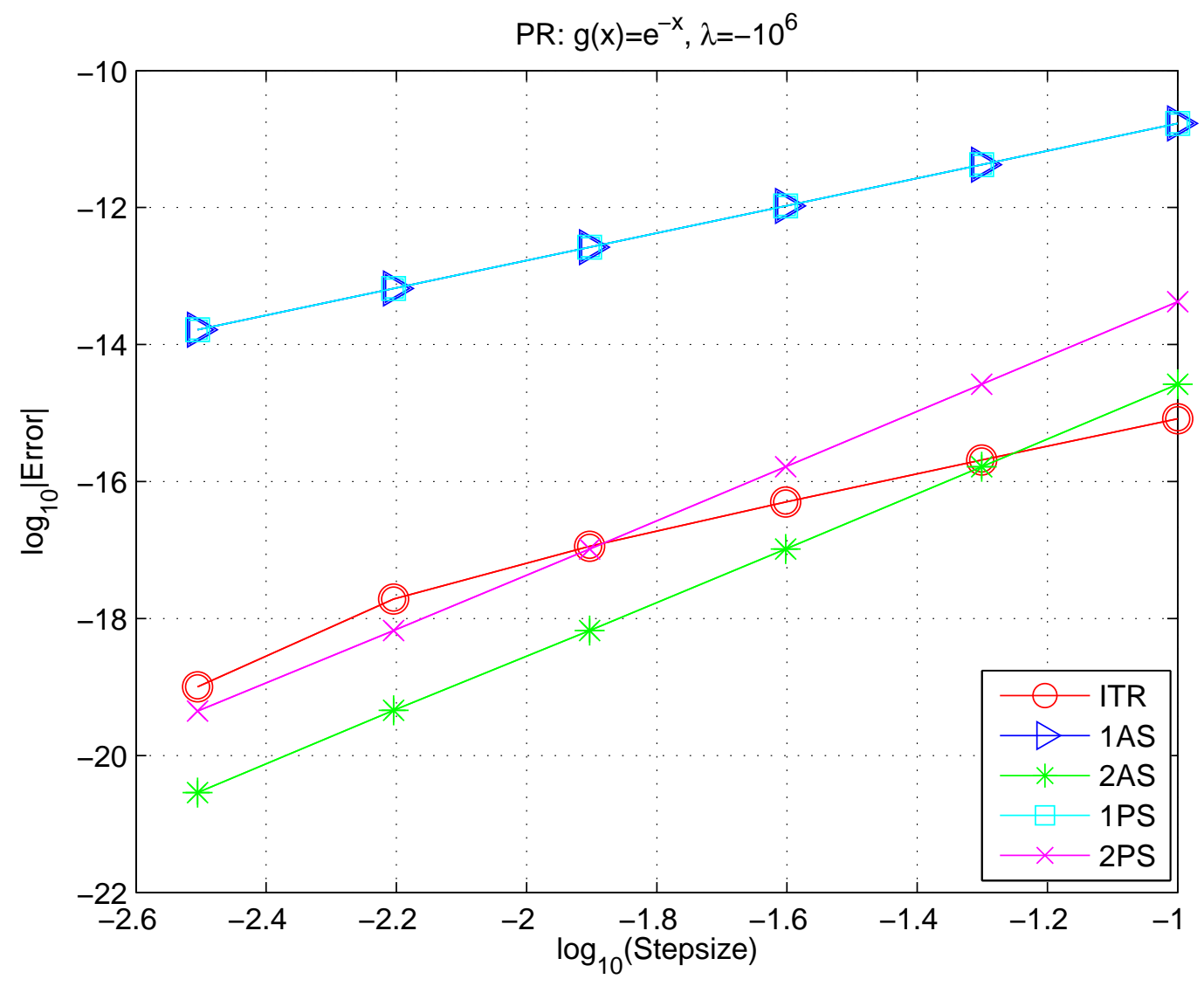

Figure 2: The PR problem order behaviour of the ITR for the stiff case. 


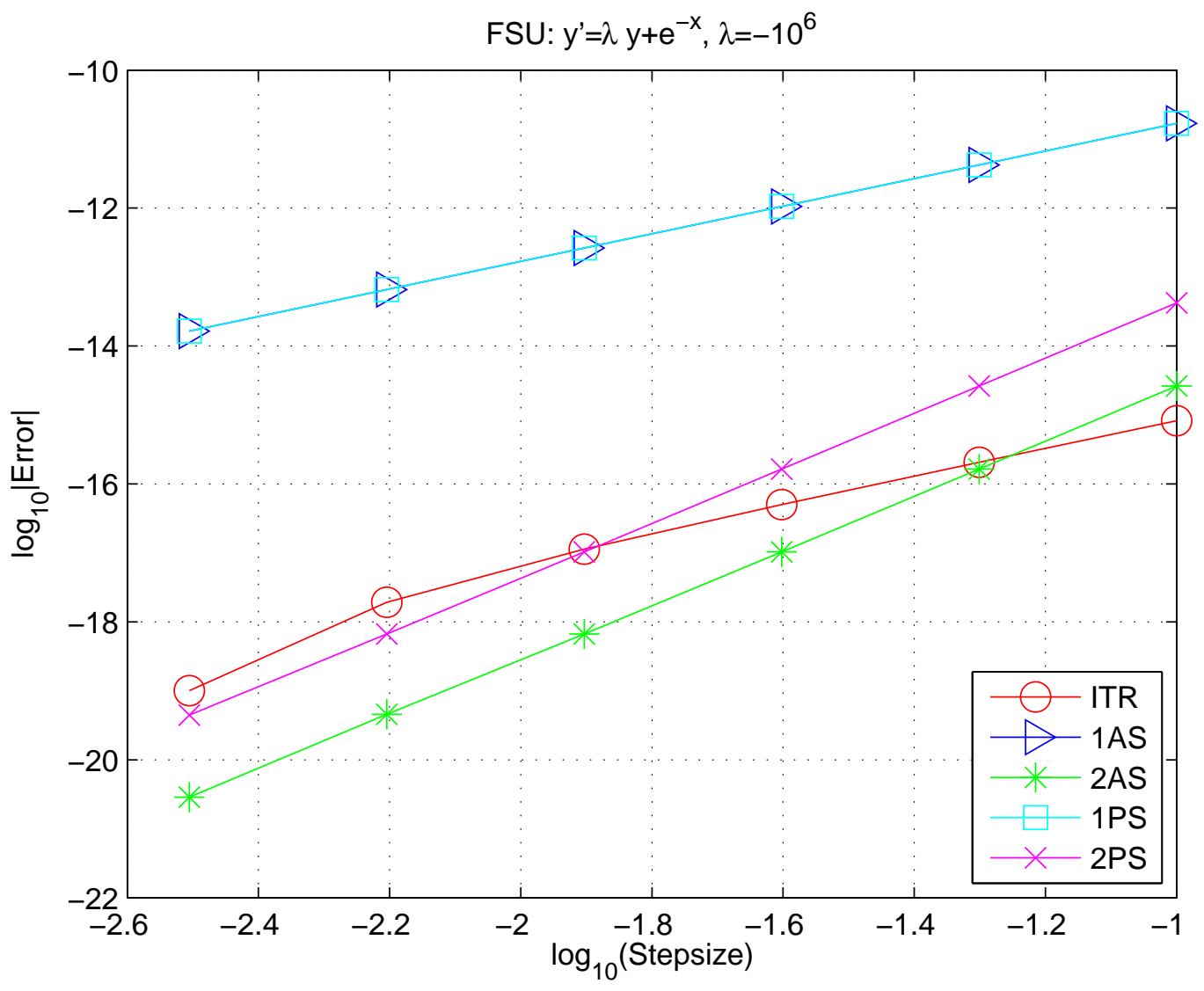

Figure 3: Order behaviour of the ITR applied to the FSU problem. 


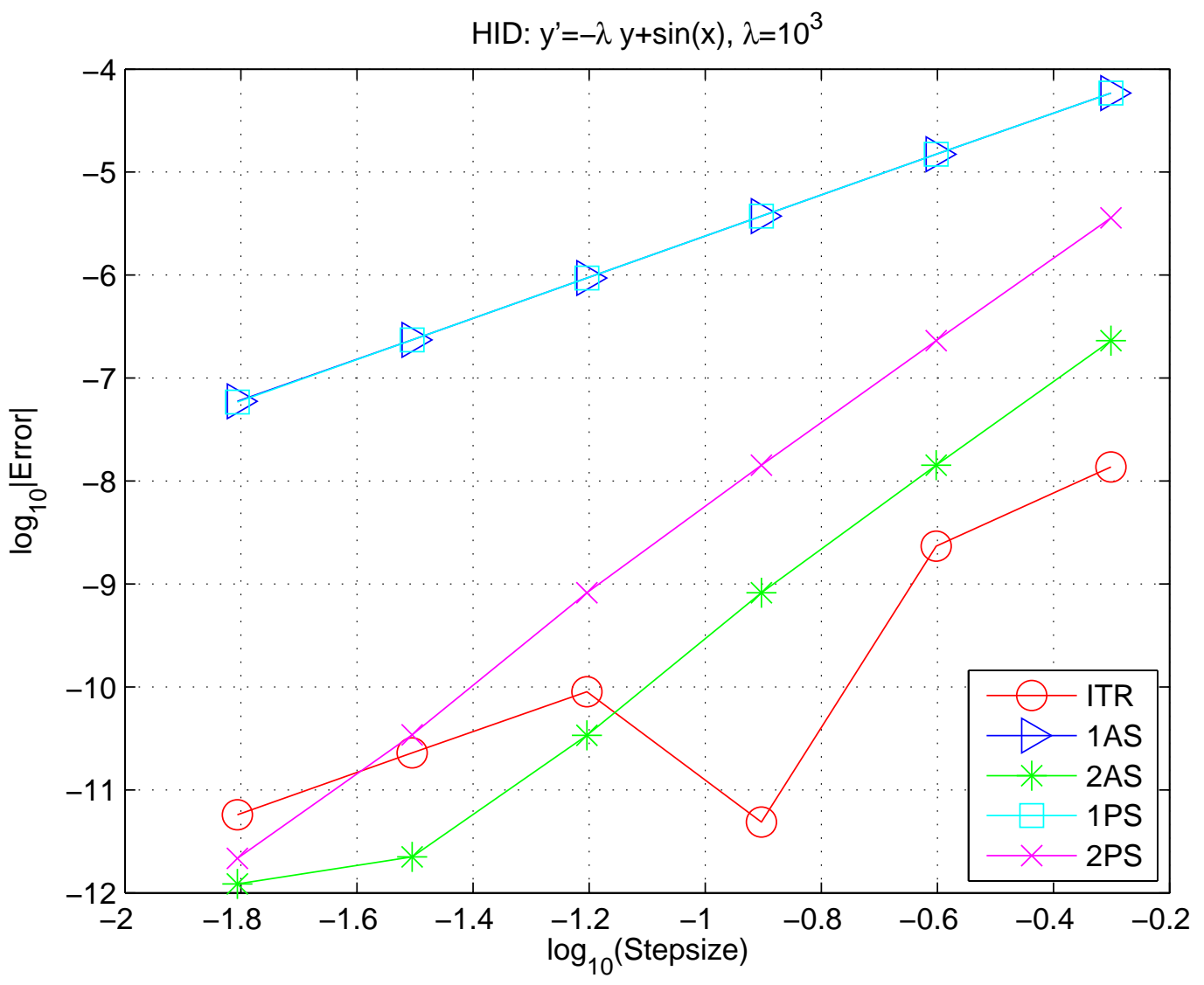

Figure 4: Order behaviour of the ITR applied to the HID problem. 


\subsection{Problems using variable step sizes}

In the case of variable step size, the step size selection is based on the standard step size controller [12] and the error estimation is obtained using symmetrization. For example, the update is $y_{n}=Y_{2}^{n}$ where $Y_{2}^{n}$ is the second internal stage value for the ITR at the nth step. The update for two-step symmetrization of the ITR is as in equation (15). The local error is

$$
\widehat{e}_{n}=\widehat{y}_{n}-y_{n} \text {. }
$$

We consider the following variable step size problems.

- The van der Pol (VDP) [8] problem

$$
y_{1}^{\prime}=y_{2}, \quad y_{2}^{\prime}=\frac{1}{\epsilon}\left[\left(1-y_{1}^{2}\right) y_{2}-y_{1}\right], \quad y_{1}(0)=2, \quad y_{2}(0)=0,
$$

integrated to $X=5$ and with $\epsilon=10^{-2}$.

- The Curtiss and Hirschfelder ( $\mathrm{CH}$ ) [6] problem

$$
y^{\prime}=-50[y-\cos (x)]
$$

integrated to $X=10$ with initial value $y(0)=1$.

\section{Results and discussion}

This section compares one and two-step symmetrization in active mode with variable step size. Figure 5 plots the solutions for the VDP problem and Figure 6 plots the step size selection for this problem. In this problem the step size for two-step symmetrization is larger than the one-step symmetrization in certain regions. Figure 7 plots the solution for the $\mathrm{CH}$ problem and Figure 7 plots the step size selection for this problem. In this problem, like the VDP problem, the step size for two-step symmetrization is larger than the one-step 


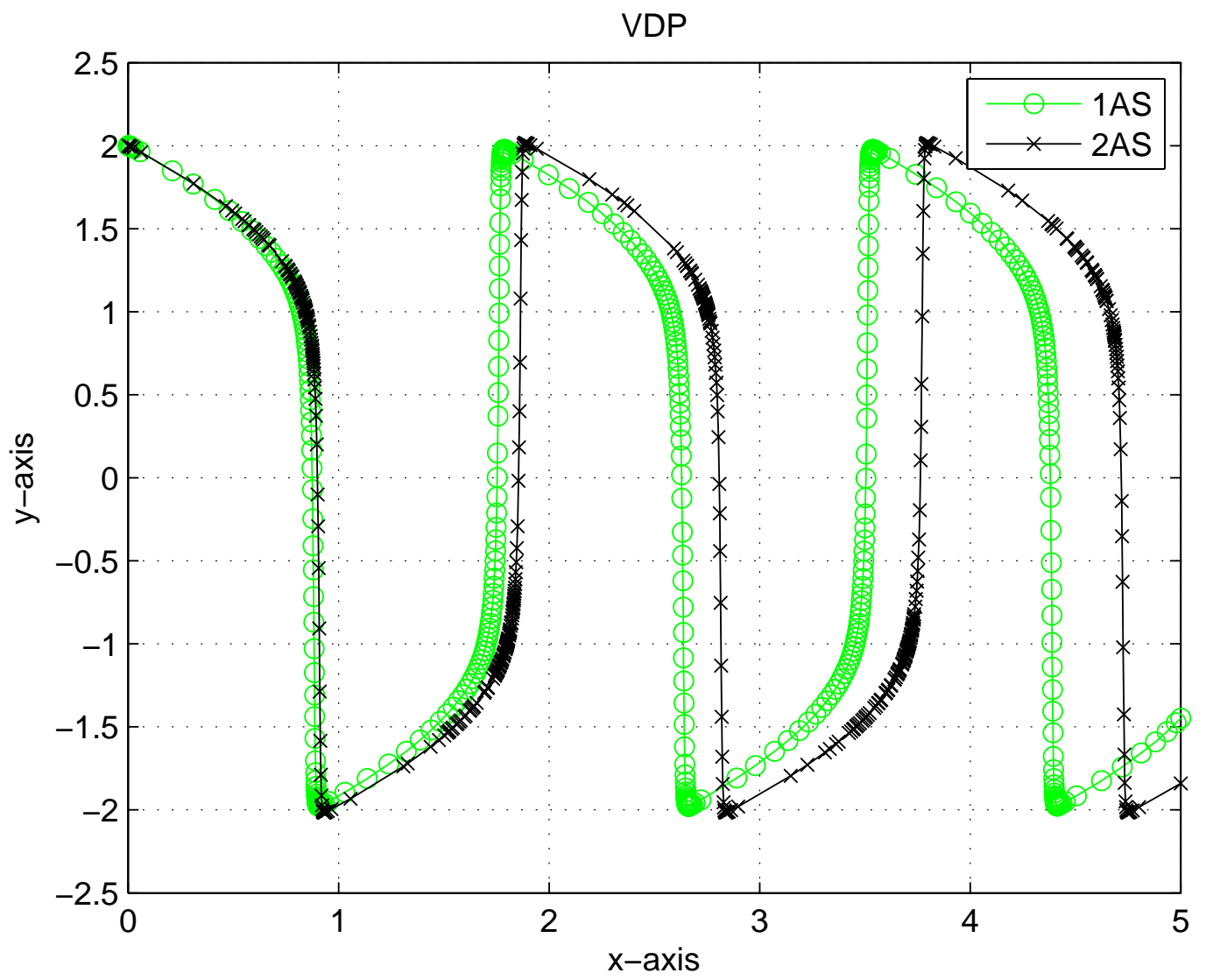

Figure 5: The VDP problem solution. 


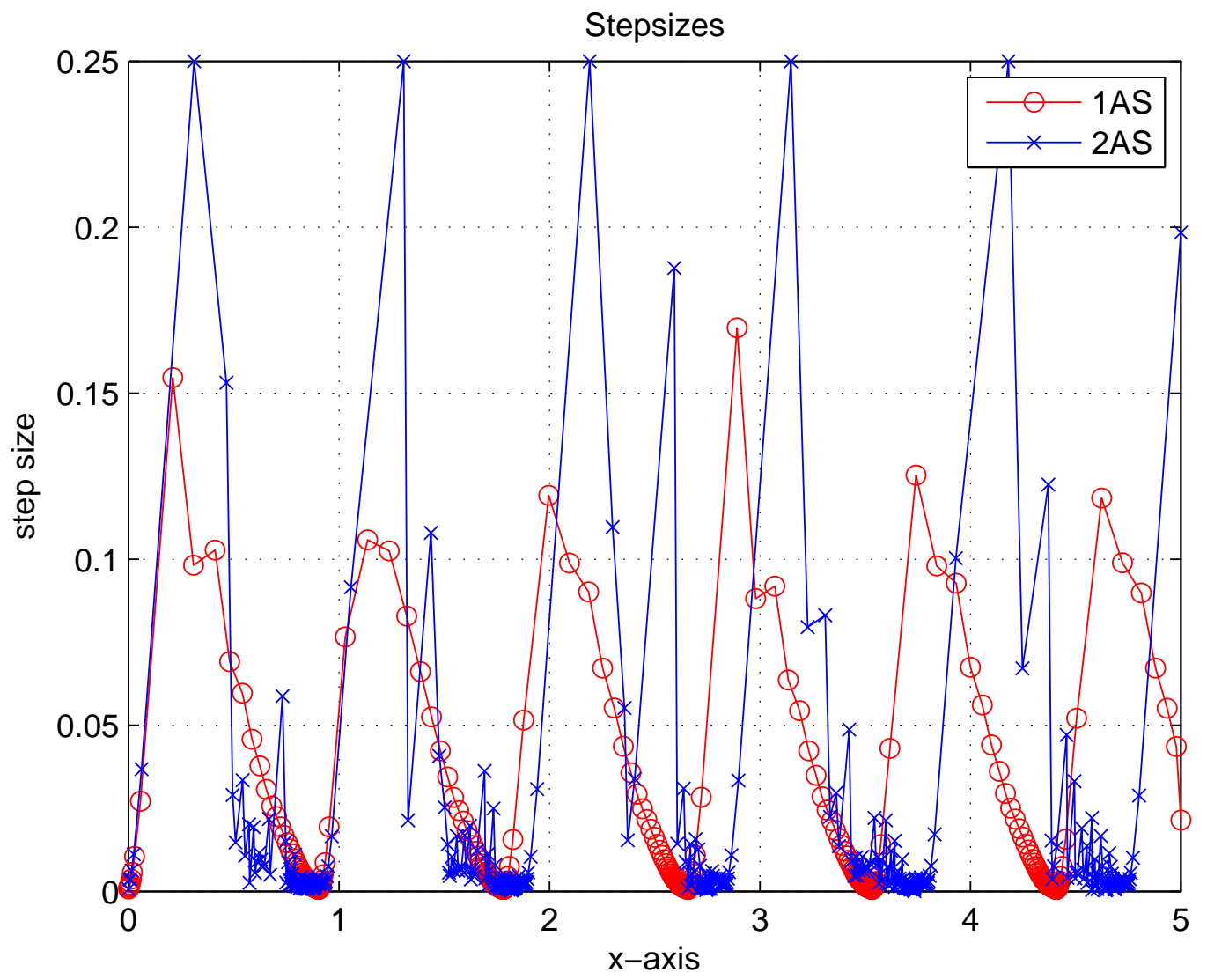

Figure 6: The VDP problem step size selection. 


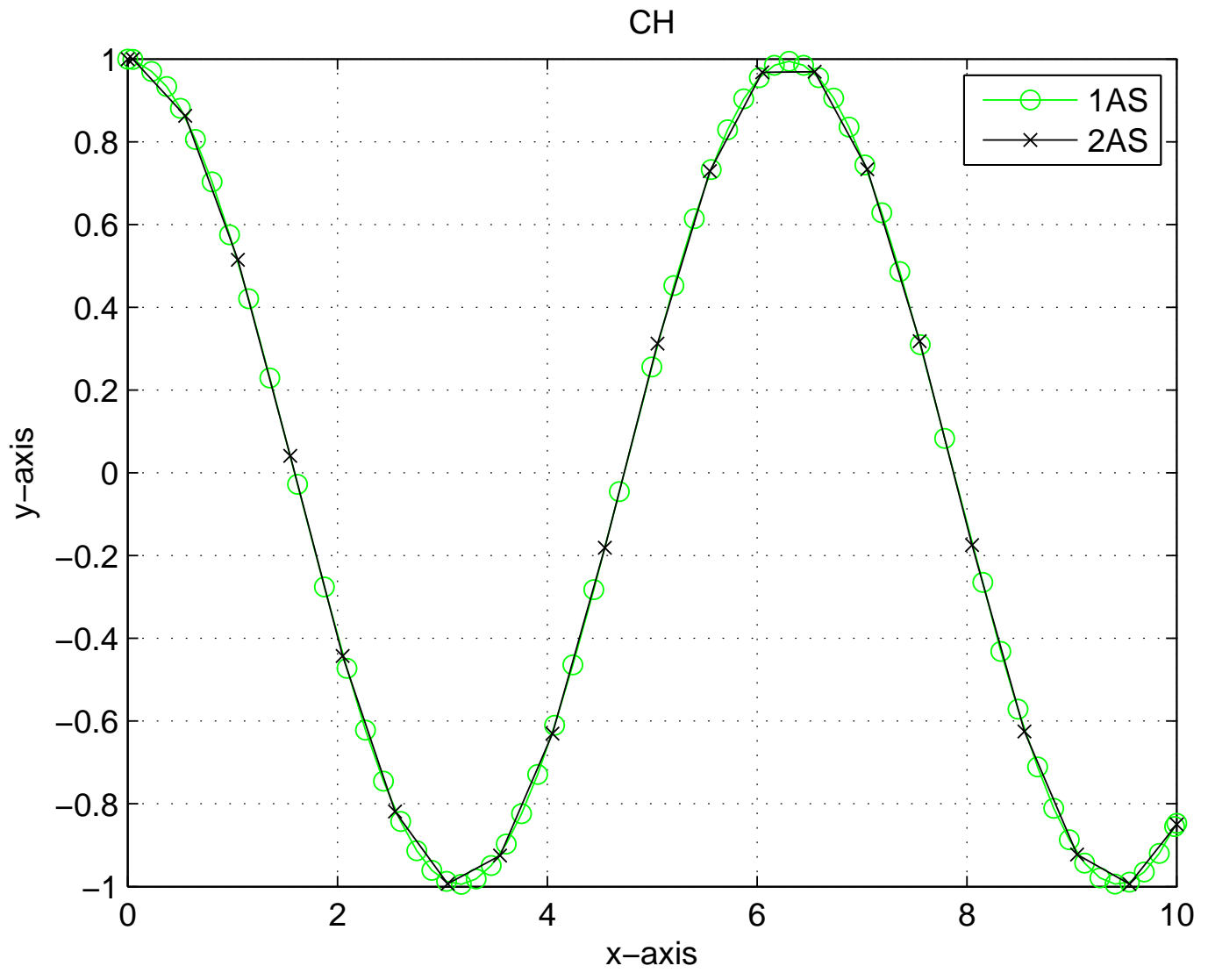

Figure 7: The $\mathrm{CH}$ problem solution. 


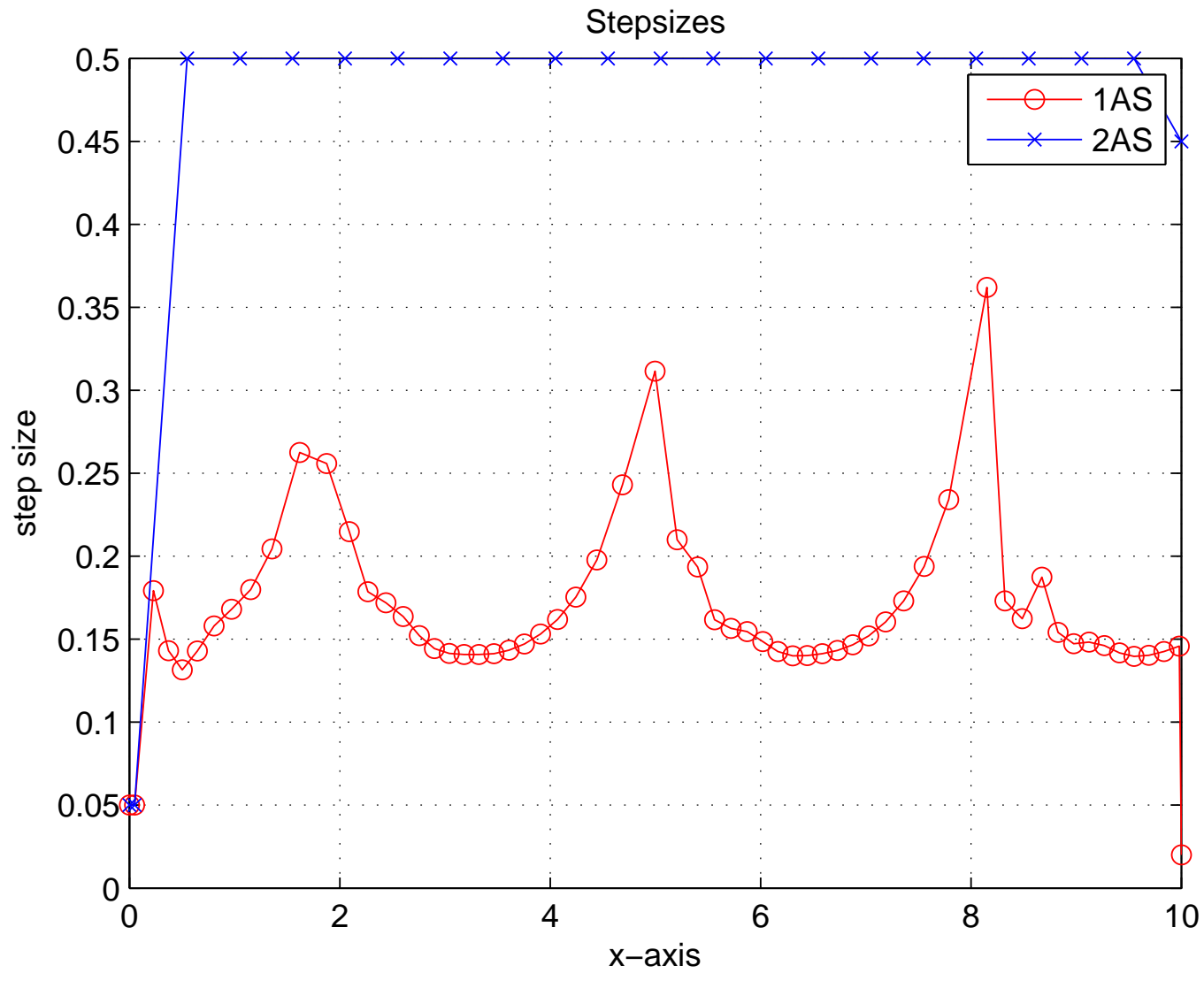

Figure 8: The $\mathrm{CH}$ problem step size selection. 
Table 1: Number of steps and error estimations.

\begin{tabular}{l|rr|rr} 
problem & steps (1AS) & steps (2AS) & error est. (1AS) & error est. (2AS) \\
\hline VDP & 612 & 911 & $4.46 \times 10^{-3}$ & $7.17 \times 10^{-4}$ \\
CH & 72 & 22 & $6.13 \times 10^{-4}$ & $4.73 \times 10^{-4}$
\end{tabular}

symmetrization. The larger step sizes result in a reduction of computation time. Figures 9 and 10 plot efficiency diagrams of CPU time versus absolute error for the VDP and $\mathrm{CH}$ problems, respectively. The plots show that the $2 \mathrm{AS}$ is more efficient than the 1AS for both problems.

To compare the accuracy, Table 1 shows the number of steps and error estimations of the one and two-step symmetrizers. For the VDP problem, 2AS requires 911 steps to get to the solution, compared to 612 steps for 1AS. Although the number of steps for 2AS are greater than for 1AS, 2AS gives better accuracy. In the $\mathrm{CH}$ problem, $2 \mathrm{AS}$ takes 22 steps which is less than $1 \mathrm{AS}$ with 72 steps, and $2 \mathrm{AS}$ is slightly more accurate than $1 \mathrm{AS}$.

\section{Conclusion}

The two-step symmetrization for the ITR was tested on five problems. With constant step size, superconvergent order four behaviour is observed. For variable step sizes, two-step symmetrization is shown to be more efficient compared to one-step symmetrization. Although some results show that the total number of steps taken are greater for two-step than one-step symmetrization, it is also shown that two-step symmetrization is more accurate than one-step symmetrization. It is of interest to apply the symmetrization to various other problems and investigate the robustness of the method. We also would like to extend the idea to higher order symmetric methods and to explore strategies for extrapolation, especially for methods that show superconvergent behaviour. 


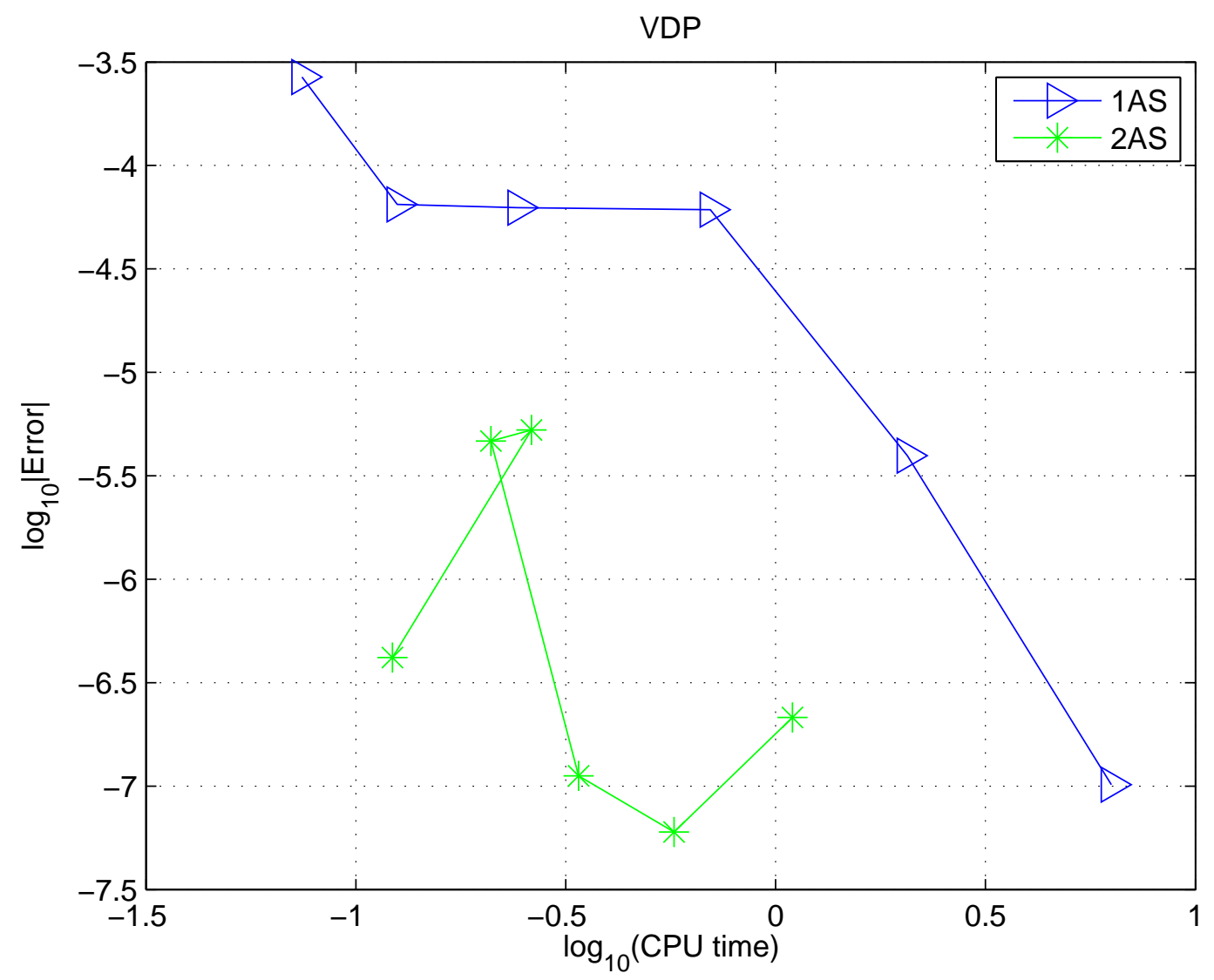

Figure 9: Efficiency diagrams for the VDP problem. 




Figure 10: Efficiency diagrams for the $\mathrm{CH}$ problem. 
Acknowledgements The work of the first author is part of her $\mathrm{PhD}$ research study in the Department of Mathematics, Faculty of Science, University of Auckland. She acknowledges support from the Press Account and a $\mathrm{PhD}$ scholarship from the Ministry of Higher Education, Malaysia and UKM, Malaysia. The authors thank the referees for their valuable comments.

\section{References}

[1] G. Bader and P. Deuflhard, A semi-implicit mid-point rule for stiff systems of ordinary differential equations, Numer. Math. 41(3):373-398, 1983. doi:10.1007/BF01418331 C543

[2] R. Bulirsch and J. Stoer, Numerical treatment of ordinary differential equations by extrapolation method, Numer. Math. 8(1):1-13, 1966. doi:10.1007/BF02165234 C543

[3] R. P. K. Chan, Extrapolation of Runge-Kutta methods for stiff initial value problems. Thesis submitted for the degree of Doctor of Philosophy at the University of Auckland, 1989.

https://researchspace.auckland.ac.nz/handle/2292/1842 C543

[4] R. P. K. Chan, A-stability of implicit Runge-Kutta extrapolations, App. Numer. Math. 22(1-3):179-203, 1996. doi:10.1016/S0168-9274(96)00031-1 C543

[5] R. P. K. Chan and N. Razali. Smoothing Effects on the IMR and ITR, Numer. Algorithms 65(3):401-420, 2013, doi:10.1007/s11075-013-9779-7 C543

[6] C. F. Curtiss, and J. O. Hirschfelder, Integration of stiff equations, $P$. Natl. Acad. Sci. 38:235-243, 1952. doi:10.1073/pnas.38.3.235 C553

[7] G. Dahlquist and B. Lindberg, On some implicit one-step methods for stiff differential equations, Royal Institute of Technology, 1973. C543 
[8] H. T. Davis, Introduction to nonlinear differential and integral equations, Dover, New York, 1962. http://catalog.hathitrust.org/Record/000619023 C553

[9] R. Frank, J. Schneid and C. W. Ueberhuber. Order results for implicit Runge-Kutta methods applied to stiff systems, SIAM. J. Numer. Analysis, 22(3):515-534, 1985. doi:10.1137/0722031 C548

[10] A. Gorgey, Extrapolation of Symmetrized Runge-Kutta Methods. Thesis submitted for the degree of Doctor of Philosophy at the University of Auckland, 2012.

https://researchspace.auckland.ac.nz/handle/2292/18996 C543

[11] W. B. Gragg, On extrapolation algorithm for ordinary initial value problems, SIAM J. Numer. Anal. 2(3):384-403 1965. doi:10.1137/0702030 C542, C546

[12] E. Hairer and G. Wanner, Solving ordinary differential equations, II. Stiff and differential-algebraic problems, Springer Series in Computational Mathematics, 14. Springer-Verlag, Berlin, 1991. doi:10.1007/978-3-662-09947-6 C544, C547, C553

[13] R. Holsapple, R. Iyer and D. Doman, Variable step-size selection methods for implicit integration schemes for ODEs, Int. J. Numer. Anal. Mod., 4(2):210-240, 2007. http://www . math . ualberta.ca/i jnam/ Volume-4-2007/No-2-07/2007-02-04.pdf C548

[14] B. Lindberg, On the smoothing and extrapolation for the trapezoidal rule, BIT, 11(1):29-52, 1971. doi:10.1007/BF01935326 C543

[15] A. Prothero and A. Robinson, On the stability and accuracy of one-step methods for solving stiff systems of ordinary differential equations, Math. Comput. 28:145-162, 1974. doi:10.1090/S0025-5718-1974-0331793-2 C544, C548 
[16] L. F. Richardson, J. A. Gaunt. The Deferred Approach to the Limit, Philos. T. R. Soc. A 226:299-361, 1927. doi:10.1098/rsta.1927.0008 C542

[17] H. J. Stetter, Analysis of Discretization Methods for Ordinary Differential Equations, Springer-Verlag, Berlin, 1973. http://www. springer.com/us/book/9783642654732 C542

\section{Author addresses}

1. N. Razali, Department of Mathematics, Faculty of Science, University of Auckland, New Zealand; Fundamental Engineering Unit, Faculty of Engineering and Built Environment, Universiti Kebangsaan Malaysia, Malaysia.

mailto:nraz865@aucklanduni.ac.nz; helyna@ukm. edu.my

2. R.P.K. Chan, Department of Mathematics, Faculty of Science, University of Auckland, New Zealand.

mailto: chan@math. auckland.ac.nz 